\title{
Dialogue Games in Multi-Agent Systems
}

\section{Peter McBurney \\ Simon Parsons}

\author{
University of Liverpool \\ City University of New York
}

\begin{abstract}
Formal dialogue games have been studied in philosophy since at least the time of Aristotle. Recently they have been applied in various contexts in computer science and artificial intelligence, particularly as the basis for interaction between autonomous software agents. We review these applications and discuss the many open research questions and challenges at this exciting interface between philosophy and computer science.
\end{abstract}

Résumé: On étudie des jeux de dialogues formels en philosophie depuis au moins le temps d'Aristote. Récemment on les a appliqués en science informatique et en intelligence artificielle, particulièrement comme base pour l'interaction entre des logicels autonomes. Nous passons ces applications en revue, répondons à diverses questions soulevées par cette recherche, et discutons des défis à relever dans cette interface passionnante entre la philosophie et la science informatique.

Keywords: formal dialogue games, multi-agent systems, autonomous software agents

\section{Introduction}

Formal dialogue games are interactions between two or more players, where each player "moves" by making utterances, according to a defined set of rules. Their history in philosophy dates at least to Aristotle (Aristotle 1928/350 BCE) and they were widely studied by philosophers in medieval times (Hamblin 1970, Spade 1979). In modern times, philosophers have used formal dialogue games to study non-deductive forms of reasoning, such as the classical fallacies (Hamblin 1970, MacKenzie 1979), and to provide a game-theoretic semantics for intuitionistic and classical logic (Lorenzen and Lorenz 1978, Barth and Krabbe 1982) and for quantum logic (Mittelstaedt 1979). These games have also found application in computational linguistics and Artificial Intelligence (AI). In linguistics, they have been used to explain sequences of human utterances (Levin and Moore 1978), with subsequent application to machine-based natural language processing and generation (Hulstijn 2000), and to human-computer interaction (Bench-Capon et al. 1991). Within artificial intelligence, they have been applied to modeling complex human reasoning, for example in legal domains (Bench- Capon et al. 2000, Prakken and Sartor 1998), and to requirements specifications for complex software projects (Finkelstein and Fuks 1989). Dialogue games differ from the games of economic game theory (Osborne and Rubinstein 1994) in that payoffs for winning or losing 
a game are not considered, and because there is no use of uncertainty measures, such as probabilities, to model the possible moves of opponents. They also differ from the abstract games recently used as a semantics for interactive computation (Abramsly 1997), in the tradition of Jaako Hintikka's game-theoretic semantics (Hintikka 1983), since these abstract games do not share the rich rule structure of dialogue games, and are not intended to have themselves a semantic interpretation involving the beliefs or actions of multiple agents.

The main application of dialogue games in Artificial Intelligence has been to the design of protocols for interaction between autonomous software entities, called agents, and our focus in this paper will be on this application. We begin, in Section 2 , with a brief introduction to the subject of autonomous agents and multi-agent systems. This is followed, in Section 3, with an overview of an influential typology of human dialogues, which will be used to classify agent interactions. Section 4 then presents a model of a formal dialogue game protocol, following which we consider, in Section 5 , several recent proposals for agent interaction protocols based on dialogue games. In Section 6, we discuss some of the many open issues current in this domain, whose resolution will require contributions from computer science, philosophy and mathematics.

\section{Agents and agent interactions}

Over the last decade, the concept of autonomous software agents has become increasingly important in Computer Science, simultaneously with the rise of largescale distributed computer systems, such as the Internet. ${ }^{1}$ There are many distinct definitions of what comprises an autonomous software agent, and as yet no consensus among computer scientists. However, for our purposes, it will suffice to use the formulation of Michael Wooldridge and Nicholas Jennings (Wooldridge and Jennings 1995). They described an autonomous software agent as being situated in a specific environment and exhibiting there four primary characteristics: autonomy of decision-making; social awareness; reactivity to events in its environment; and proactivity, as it seeks to actively achieve some goals within its environment. A multi-agent system is a community of two or more autonomous computational agents interacting. An example could be a network of water data monitors, each responsible for water and flood control in part of some geographic region. In this example, each monitor may be able to collect local information, send and receive information to and from other monitors, and take local action, such as opening a dam sluice to release excess water. Actions may be taken independently or in collaboration with other monitors, in which case dialogue between the agents might be necessary to agree to the need for, and the nature of, any such actions. Similarly, some global actions may require each agent to act locally for its desired effects to be realized.

There are two sets of design issues which currently pre-occupy researchers in this field: The design and engineering of the individual agents in a multi-agent 
system, and the design and engineering of the system itself. For the design of individual agents, the philosophy of intention, particularly the work of Michael Bratman (Bratman 1987) and John Pollock (Pollock 1995), has been influential, along with ideas from economic theory and cognitive psychology. Formalization of these approaches has often drawn heavily on modal logic, (e.g., Wooldridge 2000a).

For the design of multi-agent systems, attention has focused on the design of communications and interaction protocols. Here, several approaches have been adopted. One widespread approach has been the use of economic interaction mechanisms, such as auctions (Rosenschein and Zlotkin 1994). Typically, these have advantages of simplicity and analytical tractability; for example, one can often determine the extent to which the outcomes of an auction have desirable properties, such as Pareto-optimality (Sandholm 1999). However, the information which can be transmitted in these interactions is limited: participants in an auction, for example, cannot normally provide the reasons for their bids or the reasons for their acceptance or rejection of the bids of others. It is reasonable to suppose that the ability to present such reasons should increase the likelihood that agents participating in an interaction would reach successful resolution (Parsons, Sierra and Jennings 1998).

A second approach to communications protocol engineering, permitting greater expressiveness than do auction mechanisms, has been the design of generic agent communications languages. Here, there are two main proposals: the Knowledge Query and Manipulation Language (KQML) (Mayfield et al. 1996), which arose from work sponsored by the U.S. Government's Defense Advanced Research Projects Agency (DARPA); and the Foundation for Intelligent Physical Agents' Agent Communications Language (FIPA 2001), the FIPA ACL, which arose from attempts to develop an industry standard for agent communications. In both languages there is a two-level hierarchy for communications messages. At the lower, or "inner" level, the content of messages-the topics of discussion-may be expressed in any logical language agreed by the participants. Such content is then wrapped, at the next level, in one of a number of defined locutions. In the FIPA $\mathrm{ACL}$, for example, there are 22 of these; the inform locution, for example, has the following syntax (FIPA 2001, p. 11):

\section{(inform}

:sender (agent-identifier :name j)

:receiver (set (agent-identifier :name i))

:content "weather (today, raining)"

:language Prolog)

In this example the content of the message is "weather (today, raining)", while the locution in which this content is wrapped is inform. The syntax of the locution 
indicates that the computational language in which the message content is expressed is Prolog. The syntax also indicates that the sender of the message has agent-identifier $j$, while the intended recipient has agent-identifier $i$.

The FIPA ACL has been given an axiomatic semantics, called SL (for Semantic Language), based on speech act theory (FIPA 2001, Informative Annex A). ${ }^{2}$ Speech act theory, due originally to philosophers John Austin (Austin 1962) and John Searle (Searle 1969), considers spoken human utterances as actions, in so far as they may change the state of the world. Some utterances do this demonstrably as when countries issue declarations of war on other countries. Other utterances change the world unobservably, as when one person informs another of something he or she does not already know, and thereby causes a revision to the beliefs of the listener. Speech act theory classifies utterances according to their preconditions and effects, and has been used to provide a semantics for agent utterances, particularly in work by Philip Cohen, Hector Levesque and Raymond Perrault (Cohen and Levesque 1990, Cohen and Perrault 1979). Drawing on this work, the FIPA ACL Semantic Language SL is a modal logic formalism which defines preand post-conditions for the 22 locutions of the language in terms of the beliefs, desires and uncertain beliefs of the agents participating in a dialogue using the language. For example, the inform locution may only be uttered if the first agent believes the proposition to be true, which is thus a semantic condition for the locution. Further information regarding FIPA ACL and KQML may be found in Labrou et al. 1999 and Mayfield et al. 1996; a recent introduction to the subject of autonomous agents and multi-agent systems can be found in Wooldridge 2002 .

\section{Types of dialogues}

An influential model of human dialogues is the typology of primary dialogue types of argumentation theorists Douglas Walton and Erik Krabbe (Walton and Krabbe 1995). This categorization is based upon the information the participants have at the commencement of a dialogue (of relevance to the topic of discussion), their individual goals for the dialogue, and the goals they share. Information-Seeking Dialogues are those where one participant seeks the answer to some question(s) from another participant, who is believed by the first to know the answer(s). In Inquiry Dialogues the participants collaborate to answer some question or questions whose answers are not known to any one participant. Persuasion Dialogues involve one participant seeking to persuade another to accept a proposition he or she does not currently endorse. In Negotiation Dialogues, the participants bargain over the division of some scarce resource. Here, the goal of the dialogue-a division of the resource acceptable to all - may be in conflict with the individual goals of the participants. Participants of Deliberation Dialogues collaborate to decide what action or course of action should be adopted in some situation. Here, participants share a responsibility to decide the course of action, or, at least, they share a willingness to discuss whether they have such a shared responsibility. Note that 
the best course of action for a group may conflict with the preferences or intentions of each individual member of the group; moreover, no one participant may have all the information required to decide what is best for the group. In Eristic Dialogues, participants quarrel verbally as a substitute for physical fighting, aiming to vent perceived grievances.

This classification has been influential in argumentation theory and in the application of dialogue game protocols to multi-agent systems. However, most actual dialogue occurrences-both human and agent-involve mixtures of these dialogue types. A purchase transaction, for example, may commence with a request from a potential buyer for information from a seller, proceed to a persuasion dialogue, where the seller seeks to persuade the potential buyer of the importance of some feature of the product, and then transition to a negotiation, where each party offers to give up something he or she desires in return for something else. The two parties may or may not be aware of the different nature of their discussions at each phase, or of the transitions between phases. Instances of individual dialogue types contained entirely within other dialogue types are said to be embedded (Walton and Krabbe 1995).

\section{Formal dialogue games}

We now present a model of a generic formal dialogue game in terms of the components of its specification, taken from McBurney and Parsons 2002a. We first assume that the topics of discussion between the agents can be represented in some logical language, whose well-formed formulae are denoted by the lowercase Roman letters, $p, q, r$, etc. A dialogue game specification then consists of the following elements:

Commencement Rules: Rules that define the circumstances under which a dialogue commences.

Locutions: Rules that indicate what utterances are permitted. Typically, legal locutions permit participants to assert propositions, permit others to question or contest prior assertions, and permit those asserting propositions that are subsequently questioned or contested to justify their assertions. Justifications may involve the presentation of a proof of the proposition or an argument for it. The dialogue game rules may also permit participants to utter propositions to which they assign differing degrees of commitment, for example: one may merely propose a proposition, a speech act which entails less commitment than would an assertion of the same proposition.

Combination Rules: Rules that define the dialogical contexts under which particular locutions are permitted or not, or obligatory or not. For instance, it may not be permitted for a participant to assert a proposition $p$ and subsequently the proposition $\sim p$ in the same dialogue, without in the interim having retracted the former assertion. 
Commitments: Rules that define the circumstances under which participants express commitment to a proposition. Typically, the assertion of a claim $p$ in the debate is defined as indicating to the other participants some level of commitment to, or support for, the claim. Since the work of philosopher Charles Hamblin (Hamblin 1970), formal dialogue systems typically establish and maintain public sets of commitments, called commitment stores, for each participant; these stores are usually nonmonotonic, in the sense that participants can also retract committed claims, although possibly only under defined circumstances.

Termination Rules: Rules that define the circumstances under which a dialogue ends.

It is worth noting here that more than one notion of commitment is present in the literature on dialogue games. For example, Hamblin treats commitments in a purely dialogical sense: "A speaker who is obliged to maintain consistency needs to keep a store of statements representing his previous commitments, and require of each new statement he makes that it may be added without inconsistency to this store. The store represents a kind of persona of beliefs; it need not correspond with his real beliefs..." (Hamblin 1970, p. 257). In contrast, Walton and Krabbe (Walton and Krabbe 1995, Chapter 1) treat commitments as obligations to (execute, incur or maintain) a course of action, which they term action commitments. These actions may be utterances in a dialogue, as when a speaker is forced to defend a proposition he has asserted against attack from others; so Walton and Krabbe also consider propositional commitment as a special case of action commitment (op. cit, p. 23). As with Hamblin's treatment, such dialogical commitments to propositions may not necessarily represent a participant's true beliefs. In contrast, Munindar Singh's social semantics (Singh 2000), requires participants in an interaction to express publicly their beliefs and intentions; these expressions are called social commitments. These include both expressions of belief in propositions and expressions of intent to execute or incur future actions. ${ }^{3}$ Our primary motivation is the use of dialogue games as the basis for interaction protocols between autonomous agents. Because such agents will typically enter into these interactions in order to achieve some wider objectives, and not just for the enjoyment of the interaction itself, we believe it is reasonable to define commitments in terms of future actions or propositions external to the dialogue. In a commercial negotiation dialogue, for instance, the utterance of an offer may express a willingness by the speaker to undertake a subsequent transaction on the terms contained in the offer. For this reason, we can view commitments as semantic mappings between locutions and subsets of some set of statements expressing actions or beliefs external to the dialogue.

Dialogue game protocols have been articulated for each of the rule-governed primary types of dialogues in the typology of Walton and Krabbe: informationseeking dialogues (Walton and Krabbe 1995, Hulstijn 2000); inquiries (McBurney 
and Parsons 2001b); persuasion dialogues (Amgoud, Maudet and Parsons 2000, Dignum et al. 2000); negotiation dialogues (Amgoud, Parsons and Maudet 2000, Hulstijn 2000, McBurney et al. 2003, Sadri et al. 2001); and deliberations (Hitchcock et al. 2002). Some of these proposals are discussed in the next section. There has even been work on non-co-operation in dialogues (Gabbay and Woods 200la, 2001 b, Dunne 2002), work which may yield dialogue game protocols for eristic dialogues. However, as mentioned earlier, most real-world dialogues (whether human or agent) involve aspects of more than one of these primary types. Two formalisms have been suggested for computational representation of combinations of dialogue: the Dialogue Frames of Chris Reed (Reed 1998), which enable iterated, sequential and embedded dialogues to be represented; and our own Agent Dialogue Frameworks (McBurney and Parsons 2002a), which permit iterated, sequential, parallel and embedded dialogues to be represented. Both these formalisms are neutral with regard to the modeling of the primary dialogue types themselves, allowing the primary types to be represented in any convenient form, and allowing for types other than the six of the Walton and Krabbe typology to be included.

\section{Examples of dialogue game protocols}

We now briefly review several of the proposals for dialogue game protocols published in the autonomous agent literature. The first of these is the protocol of Leila Amgoud, Nicolas Maudet and Simon Parsons (Amgoud, Maudet and Parsons 2000). This protocol is based on James MacKenzie's philosophical dialogue game $D C$ (MacKenzie 1979), a game for two players, both subject to the same rules. $D C$ enables the participants to argue about the truth of a proposition and was designed to study the fallacy of begging the question (petitio principii, or circular reasoning). The agent interaction protocol of Amgoud, Maudet and Parsons 2000 based on $D C$ allows four distinct locutions: assert, accept, question and challenge; these can be instantiated with a single proposition, and also, for the locutions assert and accept, with a set of propositions which together constitute an argument for a proposition. Thus the participants may communicate both propositional statements and arguments about these statements, where arguments may be considered as tentative proofs (i.e., logical inferences from assumptions which may not all be confirmed). The locutions of this protocol are similar to those of $D C$ except that they do not include a locution for retraction of assertions (called withdrawal in $D C$ ). As with MacKenzie's game, the protocol of Amgoud, Maudet and Parsons 2000 establishes commitment stores which record, in full public view, the statements each participant has asserted. The syntax for this protocol has only been provided for dialogues between two participants, but could be readily extended to more agents, as the same authors did subsequently in Amgoud and Parsons 2001.

Amgoud et al. demonstrate that their system enables persuasion, inquiry and information-seeking dialogues (Amgoud, Maudet and Parsons 2000). However, as the authors note, to permit negotiation dialogues, the protocol requires additional 
locutions. These are proposed in a subsequent paper by the same authors (Amgoud, Parsons and Maudet 2000), in which three additional locutions are suggested: request, promise and refuse. In addition to instantiation with propositions and with arguments for propositions, several of these locution can also be instantiated with a two-valued function expressing a relationship between two resources. For example, the locution promise $(\mathrm{p} \vee \mathrm{q})$ indicates a promise by the speaker to provide resource $q$ in return for receiving resource $p$.

Building on the protocol of Amgoud, Parsons and Maudet 2000, Fariba Sadri, Francesca Toni and Paolo Torroni (Sadri et al. 2001) propose a similar protocol but with fewer locutions. The legal locutions proposed here are: request, promise, accept, refuse, challenge, and justify. The contents of the locutions request and promise are resources, while the contents for the other four locutions are any of the six locutions. In addition, the locution justify allows the utterance of some support for a previous locution.

The dialogue-game protocols presented in the work of Frank Dignum, Barbara Dunin-Keplicz and Rineke Verbrugge (Dignum et al. 2000, 2001) are intended to enable agents to form teams and to agree to joint intentions, respectively. For both protocols, the authors assume that one agent, an initiator or proponent, seeks to persuade others (opponents) to join a team, and that another initiator (possibly the same agent) seeks to persuade team members to adopt a group belief or intention. The team-formation dialogue is modeled as an information-seeking dialogue followed by a persuasion, while the joint-intentions-formation dialogue is modeled as a persuasion dialogue, which may include embedded negotiation dialogues. For the persuasion dialogues, the authors adapt the rigorous persuasion dialogue game of Walton and Krabbe (Walton and Krabbe 1995). This game is a formalization of a critical discussion-i.e., a rigorous persuasion-in philosophy. Such dialogues involve two parties, one seeking to prove a proposition, and one seeking to disprove it. ${ }^{4}$ Unfortunately, the authors do not specify their team-formation dialogue game models completely, for example, nowhere stating the set of locutions available to the participating agents. The protocol for joint intention formation dialogues (Dignum et al. 2001) includes seven locutions: statement, question, challenge, challenge-with-statement, question-with-statement and final remarks; these last include: "quit" and "won." The statements associated with challenges and questions may be concessions made by the speaker. The protocol for team formation dialogues (Dignum et al. 2000) may also use the same set of locutions, although this is not absolutely clear.

Finally, we briefly mention some of our own proposals for dialogue game agent interaction protocols. Firstly, in joint work with Rogier van Eijk and Leila Amgoud (McBurney et al. 2003), we have articulated a dialogue game protocol for negotiation dialogues between potential buyers and sellers of consumer durables; this work drew on a standard model of consumer decision-making taken from marketing theory (Lilien et al. 1992). Secondly, together with David Hitchcock 
(Hitchcock et al. 2002), we have presented a dialogue game protocol for deliberation dialogues, drawing on a theory of deliberative argument from the philosophy of argumentation (Wohlrapp 1998). Thirdly, in McBurney and Parsons 200lb, we articulated a dialogue game protocol for agents engaged in an inquiry dialogue. This protocol enables the participants to express uncertain beliefs about claims and to resolve these on the basis of the arguments for and against the claims presented in the dialogue, and was designed in accordance with the philosophy of science of Paul Feyerabend (Feyerabend 1971) and Marcello Pera (Pera 1994). Inquiries involve a disinterested search for the truthful answer to some question. In many instances, however, we may desire particular answers to a question, such as when we seek to identify the possible risks of a new technology. In these cases our search is overlaid with values we impose on the answer-space; in McBurney and Parsons 2001a, we proposed a dialogue game protocol for agents engaged in such an inquiry.

\section{Issues and Challenges}

The use of formal dialogue games as the basis for agent interaction protocols has only just begun, and there are many challenging issues still open. In this section we consider some of these.

\subsection{Protocol Semantics}

One of the reasons for the popularity of the FIPA ACL is the fact that it has been given a well-defined semantics (Labrou et al. 1999). This semantics, as we mentioned above, is based on speech act theory and is defined in terms of the certain beliefs, uncertain beliefs and intentions of the participating agents. Having such a defined semantics means that participants know precisely what other speakers mean and intend by their utterances, assuming those others are conforming to the semantics. However, verifying conformance to the semantics is a conceptually challenging task (Pitt and Mamdani 1999, Wooldridge 2000b), since it is always possible for a sufficiently clever agent to simulate insincerely any required semantic state.

The development of appropriate semantics for dialogue game protocols is still very immature, although several different types of semantics have been defined for these protocols. The first of these involves defining locutions in terms of the pre-conditions necessary for, and the post-conditions arising from, their utterance, i.e., what is termed an axiomatic semantics in computer programming language theory (Tennent 1991). We can distinguish, as in McBurney 2002, between two types of axiomatic semantics. In a public axiomatic semantics, the pre-conditions and post-conditions of each locution are given only in terms of observable linguistic behaviour, and so conformity with the protocol can be verified by anyone observing the dialogue. The protocols in Hitchcock et al. 2001, McBurney et 
al. 2003, McBurney and Parsons 2001a, 2001b have been given a public axiomatic semantics. A private axiomatic semantics, on the other hand, is one in which some or all locutions have pre- or post-conditions defined in terms of the internal states of the participants. The protocols of Amgoud, Maudet and Parsons 2000, Amgoud, Parsons and Maudet 2000, Sadri et al. 2001, Dignum et al. 2001 have been given such a semantics, as has the FIPA ACL (FIPA 2001). For the protocols of Amgoud, Maudet and Parsons 2000, Amgoud, Parsons and Maudet 2000, each participating agent is assumed to be vested with a private argumentation-based reasoning mechanism, which generates defeasible arguments from premises according to defined procedures, as in Dung 1995. The mechanism also permits a preference ordering over the arguments. An agent may utter a locution assert( $p)$, for a proposition $p$, only if that agent has an acceptable argument for $p$ in its own knowledge base, or in its knowledge base combined with the public commitment stores of the other participants. Here, acceptable arguments are those which survive a specified process of attack and defence within an argumentation framework (Dung 1995).

In the case of the protocol of Sadri et al. 2001, which is designed for dialogues over scarce resources (i.e, negotiation dialogues), utterances are linked to a firstorder logic describing resources. In this semantics, the knowledge of an agent is described as an abductive logic program consisting of if-then rules and of statements regarding the resources owned by the agent (op. cit. Section 3.1). Integrity constraints are placed on this knowledge in the form of rules ${ }^{5}$ which provide an agent with possible responses to utterances it receives. An example of such a rule is: Accept a request. The abducibles of this logic program are then the possible locutions which the agent may utter in response to a message it receives. For the protocol of Dignum et al. 2001, the authors assume that the participating agents have a Belief-Desire-Intention (BDI) mental architecture (Wooldridge 2000a) and vest the locutions with a private axiomatic semantics: as with the speech-act semantics of the FIPA ACL (FIPA 2001), locutions are defined in terms of their impacts on, and pre-conditions of, these private mental states.

One may also view a dialogue under a given protocol as a sequence of commands in a programming language which operates on the states of a virtual computer comprising the internal states of the agents participating in the dialogue. This view leads to the definition of an operational semantics for the protocol (Tennent 1991) in which locutions are defined in terms of their state transitions. Operational semantics have recently been defined for various agent communications languages, (e.g., van Eijk et al. 2002). To our knowledge, the only dialogue game protocol provided with an operational semantics is the consumer negotiation protocol of McBurney et al. 2003.

Programming language theory also entertains the concept of a denotational semantics (Tennent 1991), in which a translation is given between the commands in a programming language and the objects of some mathematical domain. In 
McBurney and Parsons $2002 \mathrm{~b}$, we defined a denotational semantics for protocols in terms of sub-spaces of $n$-dimensional euclidean space. In this semantics, dialogues conducted according to a given protocol are mapped to directed paths in the associated sub-space. Another denotational approach arises from viewing agents engaged in dialogue as jointly constructing meaning as the dialogue proceeds, in the same way that humans using natural language may be thought to do. Thus, there may be value in defining a denotational semantics which is constructed incrementally in the course of a dialogue, in a manner analogous to the Discourse Representation Structures of natural language semantics in linguistics (Kamp and Reyle 1993). This is a line of research we are pursuing, particularly with regard to negotiation dialogues (McBurney and Parsons 2002c).

\subsection{Formal Properties}

Why define a semantics for a protocol? One reason, mentioned above, is so that the participants share common meanings for utterances. Another reason is to enable a better understanding of the formal properties of a protocol and of the dialogues conducted under it. The study of the formal properties of dialogues and protocols is, like the development of formal semantics, still very immature, and considerable scope exists for further research in this area.

One property of great interest is termination: under what circumstances will dialogues conducted under a given protocol terminate? For instance, Sadri and her colleagues (Sadri et al. 2001) demonstrate that a dialogue under their protocol for resource negotiations will always terminate in a finite number of steps, if conducted between agents vested with the abductive logic program mechanisms described in the paper. ${ }^{6}$ Similarly, Parsons et al. (Parsons, Wooldridge and Amgoud 2002,2003) consider the termination properties of the protocols of Amgoud, Maudet and Parsons 2000, Amgoud, Parsons and Maudet 2000 for agents vested with argumentation-based decision architectures.

A set of related questions concern the computational complexity of dialogues using dialogue game protocols: How many algorithm steps are required, for the most efficient algorithm, for a participant to decide what to utter in a dialogue under a given protocol? How many algorithm steps are required, for the most efficient algorithm, to determine whether the locution a participant proposes to utter actually conforms to the protocol under which the dialogue is conducted? And, how many dialogue utterances are required for normal termination of a dialogue under a given protocol? There has been some preliminary work by computer scientists addressing these questions. In the papers just cited (Parsons, Wooldridge and Amgoud 2002, 2003) Parsons, Wooldridge and Amgoud consider the first and third questions for dialogue game protocols for information-seeking, inquiry and persuasion between agents with different degrees of scepticism regarding the information they receive. Dunne and McBurney (2002) consider the first question for general persuasion dialogue protocols, while Dunne and Bench-Capon (2001) 
consider the third question for a specific two-party, persuasion dialogue protocol. In addition, there has been related work by some of these authors, looking at the computational complexity of termination of general negotiation mechanisms (not only those involving dialogue game protocols) (Wooldridge and Parsons 2000) and the computational complexity of deciding the status of particular arguments in computational argumentation systems (Dunne and Bench-Capon 2002). ${ }^{7}$

A third property of importance in practical applications concerns the degree to which a dialogue protocol may support automated dialogues between suitablyequipped software agents, what may be called automatability. In McBurney et al. 2003, we showed, using an operational semantics, that agents vested with appropriate, domain-specific, internal decision mechanisms could undertake fully automated consumer negotiation dialogues using the dialogue game protocol we defined. The internal decision mechanisms were derived from standard models of buyer and seller decision-making taken from marketing theory (Lilien et al. 1992). No other dialogue game protocol so far proposed has been shown to be automatable.

\subsection{Protocol Design and Assessment}

One reason a more thorough study of protocol properties is needed is to provide assistance to designers and users of agent interaction protocols. At present, designers of dialogue game protocols currently have no guidance for issues such as:

$\S$ How many locutions should there be?

$\S$ What types of locutions should be included? For example: assertions, questions, etc.

$\S$ What are the appropriate rules for the combination of locutions?

$\S$ When should behavior be forbidden, e.g., repeated utterance of one locution?

$\S$ Under what conditions should dialogues be made to terminate?

$\S$ When are dialogues conducted according to a particular protocol guaranteed to terminate?

Similarly, the absence of formal studies of dialogue game protocols means that agents (or their human principals) intending to use such protocols have no guidance for issues such as:

$\S$ When are two protocols the same? With the proliferation of protocols, it will become important for agents to decide which protocol should be used for particular interactions.

$\S$ How may different protocols be compared and how may differences be measured?

$\S$ Which protocols are to be preferred under which circumstances? In other words, what are their advantages and disadvantages?

$\S$ What is the formal relationship between dialogue game protocols and general agent communications languages, e.g., FIPA $\mathrm{ACL}$. 
$\S$ When are dialogue game protocols preferable to other forms of agent inter action, such as auction mechanisms or general agent communications languages?

As part of a longer-term project to develop a formal theory of interaction protocols, we have taken initial steps towards answering some of these questions. In work with Michael Wooldridge (McBurney, Parsons and Wooldridge 2002), we proposed thirteen desirable properties of interaction protocols using dialogue games. These properties included: Separation of syntax and semantics, so that conformance with the protocol could be assessed on the basis only of observable behaviour; Clarity of argumentation theory, so that participants would know in advance their dialectical obligations when making assertions, contesting others' assertions, etc; and Enablement of self-transformation, so that participants would be able to change their beliefs, preferences or intentions, and readily express such changes, in the course of an interaction. We applied these 13 properties to assess several existing dialogue game protocols and to the FIPA ACL; all were found wanting, to a greater or lesser extent.

In another step towards a formal theory of interaction protocols we have considered when two protocols may be considered the same. Not only is an answer to this question necessary for choosing between protocols, but it is also essential in order to assess if a protocol is new (in the sense of providing a different functionality to an existing protocol rather than just having equivalent locutions with different names) or to assess if a protocol conforms to some specification (such as that laid down as the standard for interacting within some electronic institution (Rodriguez et al. 1998)). In work with Mark Johnson (McBurney, Parsons and Johnson 2002), we have recently defined several reasonable notions of protocol equivalence and shown that these lead to distinct classes of protocols. Some of these notions were derived from ideas of process equivalence (Milner 1989), and the links between protocols and processes are worth further exploration.

\section{Conclusion}

In this paper, we have provided a brief review of the application of formal dialogue games from the philosophy of argumentation to the problem of the design of protocols for interaction between autonomous software agents. These games have almost 2500 years of study in philosophy behind them, and show great promise for agent communications. This area of Artificial Intelligence still has many open questions, research challenges and implementation issues. We believe that dialogue game protocols have great potential for multi-agent systems applications because they represent an effective compromise between the strict rule-governed nature of economic auction mechanisms and the greater expressiveness of generic agent communications languages, such as the FIPA ACL. 


\section{Notes}

As so often happens in Computer Science, practice here has preceded theory (Smith 2002).

2 For a description of different types of computer program semantics, see, for example, Tennent 1991.

${ }^{3}$ It is worth noting that all these notions of commitment differ from that commonly used in discussion of the internal states of software agents, namely the idea of the persistence of a belief or an intention (Wooldridge 2002, p. 205). As Singh (1996) argues, there is a qualitative difference between social commitments of the kind discussed here, and personal commitments of the kind encoded in beliefs, desires, and intentions. He further argues that one kind of commitment cannot be derived from another.

4 Note, however, that the persuasion dialogues of Walton and Krabbe (1995) deal only with beliefs, not intentions.

5 Confusingly called dialogue protocols by these authors.

"To some extent this result is not surprising since these mechanisms require the agents to be cooperative.

${ }^{7}$ It is interesting that all the published research on the computational complexity of dialogue has been undertaken by people - Amgoud, Bench-Capon, Dunne, McBurney, Parsons and Wooldridge - who have worked, or do currently, at the University of Liverpool, UK.

\section{References}

Abramsky, S. (1997). "Semantics of interaction: an introduction to game semantics", pp. 1-31 in A. M. Pitts and P. Dybjer (Editors), Semantics and Logics of Computation. Cambridge, UK: Cambridge University Press.

Amgoud, L., N. Maudet and S. Parsons. (2000). "Modelling dialogues using argumentation" pp. 31-38 in E. Durfee (Editor): Proceedings of the Fourth International Conference on Muli-Agent Systems (ICMAS 2000). Boston, MA, USA: IEEE Press.

Amgoud, L., S. Parsons and N. Maudet. (2000). "Arguments, dialogue, and negotiation", pp. 338-342 in W. Horn (Editor): Proceedings of the Fourteenth European Conference on Artificial Intelligence (ECAI 2000). Berlin, Germany: IOS Press.

Amgoud, L. and S. Parsons. (2001). "Agent dialogues with conflicting preferences", pp. 1-14 in J-J. Meyer and M. Tambe (Editors): Pre-Proceedings of the Eighth International Workshop on Agent Theories, Architectures, and Languages (ATAL 200I). Seattle, WA, USA.

Aristotle. (1928). Topics. (W. D. Ross, Editor). Oxford, UK: Clarendon Press. (Original work published c. $350 \mathrm{BCE}$ ).

Austin, J. L. (1962). How To Do Things with Words. Oxford, UK: Oxford University Press.

Barth, E. M. and E. C. W. Krabbe. (1982). From Axiom to Dialogue: A Philosophical Study of Logics and Argumentation. Berlin, Germany: Walter de Gruyter.

Bench-Capon, T. J. M., P. E. Dunne and P. H. Leng. (1991). "Interacting with knowledgebased systems through dialogue games", pp. 123-140 in: Proceedings of the Elev. enth International Conference on Expert Systems and Applications. Avignon, France.

Bench-Capon, T. J. M., T. Geldard and P. H. Leng. (2000). "A method for the computational modelling of dialectical argument with dialogue games", Artificial Intelligence and Law, 8: 233-254. 
Bratman, M. E. (1987). Intention, Plans, and Practical Reason. Cambridge, MA, USA: Harvard University Press.

Cohen, P. R., and H. J. Levesque. (1990). "Rational interaction as the basis for communication", pp. 221-255 in: P. R. Cohen, J. Morgan and M. E. Pollack (Editors): Intentions in Communication. Cambridge, MA, USA; MIT Press.

Cohen, P. R., and C. R. Perrault. (1979). "Elements of a plan-based theory of speech acts", Cognitive Science, 3: 177-212.

Dignum, F., B. Dunin-Keplicz and R. Verbrugge. (2000). "Agent theory for team formation by dialogue", pp. 150-166 in C. Castelfranchi and Y. Lesperance (Editors): Intelligent Agents VII: Proceedings of the Seventh International Workshop on Agent Theories, Architectures, and Languages (ATAL 2000). Lecture Notes in Artificial Intelligence Volume 1986. Berlin, Germany: Springer.

-. (2001). "Creating collective intention through dialogue", Logic Journal of the IGPL, 9 (2): 305-319.

Dung, P. M. (1995). "On the acceptability of arguments and its fundamental role in nonmonotonic reasoning, logic programming and n-persons games", Artificial Intelligence, 77 : 321-357.

Dunne, P. E. (2002). Prevarication in Dispute Protocols. Technical Report No. ULCS02-025. Liverpool, UK: Department of Computer Science, University of Liverpool.

Dunne, P. E., and T. J. M. Bench-Capon. (2001). Two Party Immediate Response Disputes; Properties and Efficiency. Technical Report No. ULCS-01-005. Liverpool, UK: Department of Computer Science, University of Liverpool.

-. (2002). "Coherence in finite argument systems", Artificial Intelligence, 141 (1-2): 187-203.

Dunne, P. E., and P. McBurney. (2002). Optimal Utterances in Dialogue Protocols. Technical Report No. ULCS-02-028. Liverpool, UK: Department of Computer Science, University of Liverpool.

van Eijk, R., F. S. de Boer, W. van der Hoek and J-J. Ch. Meyer. (2002). "A fully abstract model for the exchange of information in multi-agent systems", Theoretical Computer Science, in press.

Feyerabend, P. (1993). Against Method. London, UK: Verso. $3^{\text {td }}$ ed.; $1^{\text {st }}$ ed., 1971.

Finkelstein, A. and H. Fuks. (1989). "Multi-party specification", In: Proceedings of the Fifth International Workshop on Software Specification and Design. Pittsburgh, PA, USA: ACM Sigsoft Engineering Notes.

FIPA. (2001). Communicative Act Library Specification. Report No. XC00037H. Foundation for Intelligent Physical Agents (FIPA). 10 August 2001. Available from: www. fipa.org.

Gabbay, D. M., and J. Woods. (2001a). "Non-Cooperation in Dialogue Logic", Synthese, 127 (1-2): 161-186.

-. (2001b). "More on non-cooperation in Dialogue Logic", Logic Journal of the IGPL, $9(2): 321-339$.

Hamblin, C. L. (1970). Fallacies. London, UK: Methuen.

Hintikka, J. (1983). The Game of Language: Studies in Game-Theoretical Semantics and Its Applications. Synthese Language Library, Volume 22. Dordrecht, The Netherlands: D. Reidel. 
Hitchcock, D., P. McBurney and S. Parsons (2002). "A Framework for Deliberation Dialogues", In: H. V. Hansen, C. W. Tindale, J. A. Blair, R. H. Johnson and R.C. Pinto (Eds.). Argumentation and its Applications. Windsor, ON: OSSA. [CD-ROM]

Hulstijn. J. (2000). Dialogue Models for Inquiry and Transaction. PhD Thesis. Universiteit Twente, Enschede, The Netherlands.

Kamp, H. and U. Reyle. (1993). From Discourse to Logic: Introduction to Modeliheoretic Semantics of Natural Language, Formal Logic and Discaurse Representation Theory. Dordrecht, The Netherlands: Kluwer. Two Volumes.

Labrou, Y., T. Finin and Y. Peng. (1999). "Agent communication languages: The current landscape", IEEE Intelligent Systems, 14 (2): 45-52.

Levin, J. A. and J. A. Moore. (1978). "Dialogue-Games: metacommunications structures for natural language interaction", Cognitive Science, 1 (4): 395-420.

Lilien, G. L., P. Kotler and K. S. Moorthy. (1992), Marketing Models. Englewood Cliffs, NJ, USA: Prentice-Hall.

Lorenzen, P., and K. Lorenz. (1978). Dialogische Logik. Darmstadt, Germany: Wissenschaftliche Buchgesellschaft.

MacKenzie. J. D. (1979). "Question-begging in non-cumulative systems", Journal of Philosophical Logic, 8: 117-133.

Mayfield, J., Y. Labrou and T. Finin. (1996). "Evaluating KQML as an Agent Communication Language", pp. 347-360 in M. J. Wooldridge, J. P. Muller and M. Tambe (Editors): Intelligent Agents II. Lecture Notes in Artificial Intelligence Volume 1039. Berlin, Germany: Springer.

McBurney, P. (2002). Rational Interaction. PhD Thesis. Department of Computer Science, University of Liverpool, Liverpool, UK.

McBurney, P., R. M. van Eijk, S. Parsons and L. Amgoud. (2003). "A dialogue-game protocol for agent purchase negotiations", Journal of Autonomous Agents and Mult-Agent Systems, In press.

McBurney, P. and S. Parsons. (2001a). "Chance Discovery using dialectical argumentation", pp. $414-424$ in T. Terano, T. Nishida, A. Namatame, S. Tsumoto, Y. Ohsawa, and T. Washio (Editors): New Frontiers in Artificial Intelligence: Joint JSAI 2001 Workshop Post Proceedings. Lecture Notes in Artificial Intelligence volume 2253. Berlin, Germany: Springer.

-. $(2001 \mathrm{~b})$. "Representing epistemic uncertainty by means of dialectical argumentation", Annals of Mathematics and Artificial Intelligence, 32 (1-4): 125-169.

- (2002a). "Games that agents play: a formal framework for dialogues between autonomous agents", Journal of Logic, Language and Information, 11 (3): 315-334.

-. (2002b). "A geometric semantics for dialogue-game protocols for autonomous agent interactions", Electronic Notes in Theoretical Computer Science, 52 (2).

- (2002c). Posit Spaces: A Performative Model of e-Commerce. Technical Report No. ULCS-02-026. Liverpool, UK: Department of Computer Science, University of Liverpool.

McBurney, P., S. Parsons and M. W. Johnson. (2002). "When are two protocols the same?", In: M. P. Huget, F. Dignum and J. L. Koning (Editors): Agent Communications Languages and Conversation Policies. Proceedings of a Workshop at the 
First International Joint Conference on Autonomous Agents and Multi-Agent Systems (AAMAS 2002). Bologna, Italy.

McBurney, P., S. Parsons and M. Wooldridge. (2002). "Desiderata for agent argumentation protocols", pp. 402-409 in C. Castelfranchi and W. L. Johnson (Editors): Proceedings of the First International Joint Conference on Autonomous Agents and Multi-Agent Systems (AAMAS 2002). New York City, NY, USA: ACM Press.

Milner, R. (1989). Communication and Concurrency. Hemel Hempstead, UK: Prentice Hall.

Mittelstaedt, P. (1979). Quantum Logic. Dordrecht, The Netherlands: D. Reidel.

Osborne, M. J. and A. Rubinstein. (1994). A Course in Game Theory. Cambridge, MA, USA : MIT Press.

Parsons, S., C. Sierra and N. R. Jennings. (1998). "Agents that reason and negotiate by arguing", Journal of Logic and Computation, 8 (3): 261-292.

Parsons, S., M. Wooldridge and L. Amgoud. (2002). "An analysis of formal interagent dialogues", pp. 394401 in C. Castelfranchi and W. L. Johnson (Editors): Proceedings of the First International Joint Conference on Autonomous Agents and MuliAgent Systems (AAMAS 2002). New York, NY, USA: ACM Press.

- (2003). "Properties and complexity of some formal inter-agent dialogues", Journal of Logic and Computation, in press.

Pera, M. (1994). The Discourses of Science. Chicago, IL, USA: University of Chicago Press.

Pitt, J., and A. Mamdani. (1999). "Some remarks on the semantics of FIPA's Agent Communications Language", Joumal of Autonomous Agents and Multi-Agent Systems, 2: 333-356.

Pollock, J. L. (1995). Cognitive Carpentry: A Blueprint for How to Build a Person. Cambridge, MA, USA: MIT Press.

Prakken, H. and G. Sartor. (1998). "Modelling reasoning with precedents in a formal dialogue game", Artificial Intelligence and Law, 6: 231-287.

Reed, C. (1998). "Dialogue frames in agent communications", pp. 246-253 in Y. Demazeau (Editor): Proceedings of the Third International Conference on Multi-Agent Systems (ICMAS-98). IEEE Press.

Rodriguez, J. A., F. J. Martin, P. Noriega, P. Garcia and C. Sierra. (1998), "Towards a testbed for trading agents in electronic auction markets", AI Communications, 11 (1): 5 19.

Rosenschein, J. S., and G. Zlotkin. (1994). Rules of Encounter: Designing Conventions for Automated Negotiation among Computers. Cambridge, MA, USA: MIT Press.

Sadri, F., F. Toni and P. Torroni. (2001). "Logic agents, dialogues and negotiation: an abductive approach", in M. Schroeder and K. Stathis (Editors): Proceedings of the Symposium on Information Agents for E-Commerce, Artificial Intelligence and the Simulation of Behaviour Conference (AISB-200I). York, UK: AISB.

Sandholm, T. W. (1999). "Distributed rational decision making", pp. 201-258 in G. Weiss (Editor): Multiagent Systems: A Modern Introduction to Distributed Artificial Intelligence. Cambridge, MA, USA: MIT Press.

Searle, J. (1969). Speech Acts: An Essay in the Philosophy of Language. Cambridge, UK: Cambridge University Press. 
Singh, M. P. (1996). A Conceptual Analysis of Commitments in Multiagent Systems. type Technical Report No. 96-09. Raleigh, NC, USA: Department of Computer Science, North Carolina State University.

-. (2000). "A social semantics for agent communications languages", pp. 75-88 in F. Dignum, B. Chaib-draa and H. Weigand (Editors): Proceedings of the Workshop on Agent Communication Languages, International Joint Conference on Artificial Intelligence (IJCAI-99). Seattle, WA, USA.

Smith, B. C. (2002). "The foundations of computing", pp. 23-58 in M. Scheutz (Editor); Computationalism: New Directions. Cambridge, MA, USA: MIT Press.

Spade, P. V. (1979). "Recent research on medieval logic", Synthese, 40: 3-18.

Tennent, R. D. (1991). Semantics of Programming Languages. Hemel Hempstead, UK: Prentice-Hall.

Walton, D. N. and E. C. W. Krabbe. (1995). Commitment in Dialogue: Basic Concepts of Interpersonal Reasoning. Albany, NY, USA: State University of New York Press.

Wohlrapp, H. (1998). "A new light on non-deductive argumentation schemes", Argumentation, 12: 341-350.

Wooldridge, M. J. (2000a). Reasoning about Rational Agents. Cambridge, MA, USA: MIT Press.

-. (2000b). "Semantic issues in the verification of agent communication languages", Journal of Autonomous Agents and Multi-Agent Systems, 3 (1): 9-31.

-. (2002). Introduction to Multiagent Systems. New York, NY, USA: John Wiley and Sons.

Wooldridge, M. J. and N. R. Jennings. (1995). "Intelligent Agents: Theory and Practice", Knowledge Engineering Review, 10 (2): 115-152.

Wooldridge, M. J. and S. Parsons. (2000). "Languages for negotiation", pp. 393-397 in W. Horn (Editor): Proceedings of the Fourteenth European Conference on Artificial Intelligence (ECAI 2000). Berlin, Germany: IOS Press.

Peter McBurney Dept of Computer Science University of Liverpool Liverpool, L69 $7 Z F, U K$

p.j.mcburney@csc.liv.ac.uk

Simon Parsons Dept. of Computer \& Information Science Brooklyn College, City University of New York Brooklyn, NY 11210, USA 